\title{
Complex Flocculation of Biofilm-forming Bacteria in the Presence of Flocculating Bacteria Isolated from Activated Sludge
}

\author{
Chigusa Okano ${ }^{1}$, Koki Chiba ${ }^{2}$, Eri Nasuno ${ }^{2}$, Ken-ichi Iimura ${ }^{2}$ and Norihiro Kato ${ }^{2 *}$ \\ ${ }^{1}$ Creative Department for Innovation, Collaboration Center for Research and Development, Utsunomiya University, 7-1-2 Yoto, \\ Utsunomiya, Tochigi 321-8585, JAPAN, \\ ${ }^{2}$ Department of Material and Environmental Chemistry, Graduate School of Engineering, Utsunomiya University, 7-1-2 Yoto, \\ Utsunomiya, Tochigi 321-8585, JAPAN \\ * Corresponding author: Fax: 81-28-689-6154, e-mail: katon@cc.utsunomiya-u.ac.jp
}

\begin{abstract}
We demonstrated that complex flocculation of biofilm-forming Pseudomonas aeruginosa PAO1 and Bacillus cereus SM-5 occurred in the presence of floc-forming Pseudomonas oryzihabitans AO-363 isolated from activated sludge. Laser diffraction analysis showed a drastic decrease in the detected frequency of planktonic PAO1 cells $(1.3 \mu \mathrm{m})$ after they were mixed with AO-363 flocs. In addition, the most frequent diameter of SM-5 flocs shifted from 152 to $175 \mu \mathrm{m}$ after mixing with the AO-363 culture. Complex flocculation was also supported by analysis of the bacterial composition of the floc fraction obtained by low-speed centrifugation. Such complex microbial flocs could contribute to the development of biofilms on polystyrene surfaces. When AO-363 was co-cultured with PAO1 or SM-5, the amount of biofilm formation increased by 3-4 times compared to that in the absence of the floc-forming AO-363 cells. These results suggest that microbial flocs containing extracellular polymeric substances (EPS) could increase the development of biofilms that contain bacterial colonies and the EPS components.

Key words: biofilm formation, complex microbial flocs, bacterial flocculation, extracellular polymeric substances
\end{abstract}

\section{INTRODUCTION}

Many bacteria form a biofilm as a survival strategy to secure nutrients and also to avoid drying by limiting diffusion [1-3]. A biofilm is a low-elasticity, hydrogel-like aggregate that is regarded as a cause of periodontal disease [4], infection [5,6], and membrane biofouling [7]. Biofilm formation on bacterial cell separation membranes can reduce the membrane permeation rate, resulting in lowered performance of the wastewater treatment plant [8].

While biofilms have unfavorable effects $[9,10]$, they are also expected to have positive applications, for example, in the bioremediation of certain biotopes and natural lakes [11]. The biofilm is a colony of microorganisms that forms on aquatic plants in marshlands or on stones in artificial ponds, where they can purify water. Furthermore, biofilms have applications in agriculture as microbial pesticides [12]. By forming biofilms on leaves or stems, some harmless bacteria can prevent attachment of and infection caused by phytopathogenic bacteria.

To utilize the functional properties of biofilms for beneficial applications, attachment of the biofilm to various artificial and biological surfaces is needed. Besides adhesiveness, physical properties of the biofilm such as durability and water content are also important factors to consider. Accordingly, novel, effective methods are desired for easy immobilization of bacterial cells on specific surfaces, and also for ensuring the timely formation of biofilms composed of desired bacteria.

The process of biofilm development consists of initial bacterial adhesion on a solid surface, followed by micro-colony formation, depending on secreted molecules such as proteins and extracellular polymeric substances (EPS). On the other hand, many bacterial flocs predominantly found in complex microbial communities are utilized for sedimentation of bacteria in the activated sludge process [13,14], wherein the flocs possess components similar to those of the biofilm $[15,16]$. Therefore, we focused on biofilm development in the presence of microbial flocs containing biofilm components.

Many floc-forming bacteria have been isolated from activated sludge from treated domestic sewage and industrial wastewater [17]. Furthermore, the existence of non-floc-forming bacteria in such microbial flocs has been reported [18]. Microbial flocs are spontaneously formed from floc-forming bacteria and other planktonic cells. In this study, complex microbial flocs composed of floc-forming bacteria and nonfloc-forming, biofilm-forming bacteria were characterized for their size distribution and for their ability to induce biofilms on a polystyrene surface. In addition, we discussed the effect of the complex microbial flocs on biofilm formation, when mixing the two different flocculating bacteria with and without biofilm-forming ability. 


\section{EXPERIMENTAL METHOD}

2.1 Formation of complex microbial flocs Bacillus cereus SM-5 and Pseudomonas aeruginosa PAO1 [19] were used as biofilm-forming bacteria with and without flocculation ability, respectively. Pseudomonas oryzihabitans AO-363 is a floc-forming bacterium isolated from the activated sludge of a wastewater treatment plant in Tochigi, Japan. We tested the ability of AO-363 to form microbial complex flocs with the two biofilm-forming bacteria.

Both biofilm-forming bacteria were cultured in half-strength Luria-Bertani (LB) liquid medium with shaking for $16 \mathrm{~h}$ at $30^{\circ} \mathrm{C}$. Cell density was adjusted to $\mathrm{OD}_{600}=0.2$ by dilution with fresh half-strength LB medium. The AO-363 strain was cultured under the same conditions and the cell density of the culture broth was adjusted to OD 600 $=0.2$ or 0.6 . The diluted culture broths of PAO1 and AO-363 were mixed in a 1:1 ratio by volume and gently shaken at $160 \mathrm{rpm}\left(30^{\circ} \mathrm{C}\right)$ for $1 \mathrm{~h}$ to investigate changes in floc size due to the interaction between the cells of the two strains. The changes in size of the complex microbial flocs were also studied when the flocculating bacteria SM-5 and AO-363 were mixed.

\subsection{Characterization of complex microbial flocs}

Morphology of the planktonic cells and microbial flocs was observed by digital or optical microscope (MS-200, Asahikogaku, Axio imager M2m, Carl Zeiss). Size distributions of suspended cells and flocs were determined based on laser diffraction particle size distribution analysis (LA-300, Horiba).

Low-speed centrifugation $(30 \times g, 20 \mathrm{~min})$ of the cell suspension was used to separate the floc fraction from planktonic cells. The floc fraction was re-suspended in distilled water. The flocs were disrupted by vortexing and sonication. To investigate the composition of the complex microbial flocs, the diluted cell suspension was inoculated on LB agar plates. After incubation at $30^{\circ} \mathrm{C}$, two types of colonies with different morphologies appeared and were counted. Respective colonies could be counted by plating out diluted floc fractions to the LB agar plate. The ratio of the abundances of floc-forming bacteria and biofilm-forming bacteria in the complex microbial flocs was thus determined.

2.3 Effects of the complex microbial flocs on biofilm formation

To promote biofilm formation, the suspension of the complex microbial flocs was gently poured into a 6 -well polystyrene plate. Static culture was carried out for $24 \mathrm{~h}$ at $30^{\circ} \mathrm{C}$. The biofilm that formed on the bottom of the well was gently washed with distilled water twice and then stained with $0.1 \%$ crystal violet $(\mathrm{CV})$ aqueous solution for $20 \mathrm{~min}$ to observe the morphology using a real color confocal microscope (Optelics C130, Lasertec). After washing out the
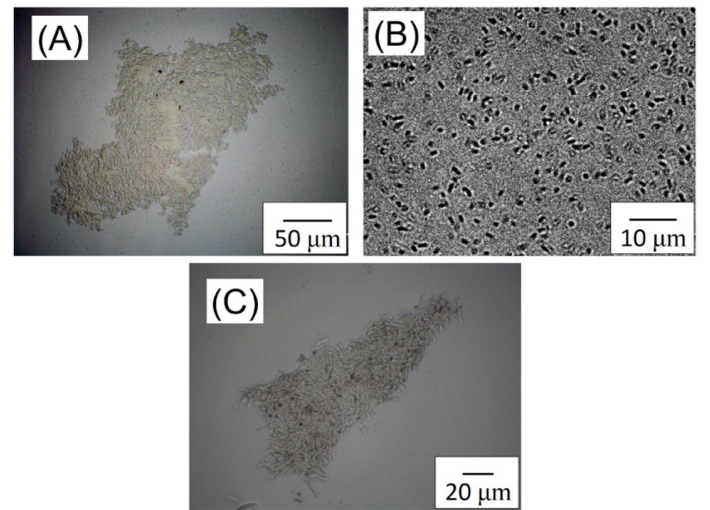

Fig. 1. Microscope images of (A) P. oryzihabitans AO-363, (B) P. aeruginosa PAO1, (C) B. cereus SM-5.

excess $\mathrm{CV}$ on the polystyrene surface, the amount of the remaining $\mathrm{CV}$ is correlated with the amount of biofilm [20]. For quantitative determination of the biofilm, the $\mathrm{CV}$ was eluted with ethanol for $1 \mathrm{~h}$ and quantified as absorbance at $595 \mathrm{~nm}\left(\mathrm{~A}_{595}\right)$.

\section{RESULTS AND DISCUSSION}

3.1 Characterization of the microbial flocs

Microscopy images of floc-forming $P$. oryzihabitans AO-363 clearly showed aggregated flocs in addition to planktonic cells (Fig. 1A), while most cells of $P$. aeruginosa PAO1 existed as planktonic cells (Fig. 1B). Most of the SM-5 cells formed dense flocs similar to those of AO-363. Formation of complex microbial flocs was studied by gently shaking AO-363 flocs with planktonic PAO1 cells or with flocculating SM-5 cells, both of which have biofilm-forming abilities.

The size distributions of cells in the mixed cultures were determined by laser diffraction analysis (Fig. 2). After optical axis adjustment, AO-363 culture broth shaken with or without the same volume of PAO1 or SM-5 was added to the fresh medium in a batch cell to measure the size distribution of the suspension $(0.1-600 \mu \mathrm{m})$ using a $650-\mathrm{nm}$ laser diode $(5 \mathrm{~mW})$. The PAO1 single culture showed a single peak with approximately $1.3 \mu \mathrm{m}$ as the most frequent diameter. This implies that most PAO1 cells were planktonic during cell growth and their aggregates were negligible (Fig. 2A). The AO-363 strain showed 2 peaks at 1.5 and $68 \mu \mathrm{m}$, corresponding to planktonic cells and microbial flocs, respectively (Fig. 2B). After 1:3 mixing of PAO1 $\left(\mathrm{OD}_{600}=\right.$ $0.2)$ and $\mathrm{AO}-363\left(\mathrm{OD}_{600}=0.6\right)$, a peak pattern relatively similar to that of AO-363 appeared (Fig. 2C) without drastic increase in the peak at around 1-2 $\mu \mathrm{m}$. These results suggest the uptake of PAO1 cells into the AO-363 flocs.

Formation of complex microbial flocs of SM-5 and AO-363 was also investigated. SM-5 single culture showed broad peaks corresponding to diameters of approximately 30 and $152 \mu \mathrm{m}$, indicating microbial flocculation (Fig. 2D). The 


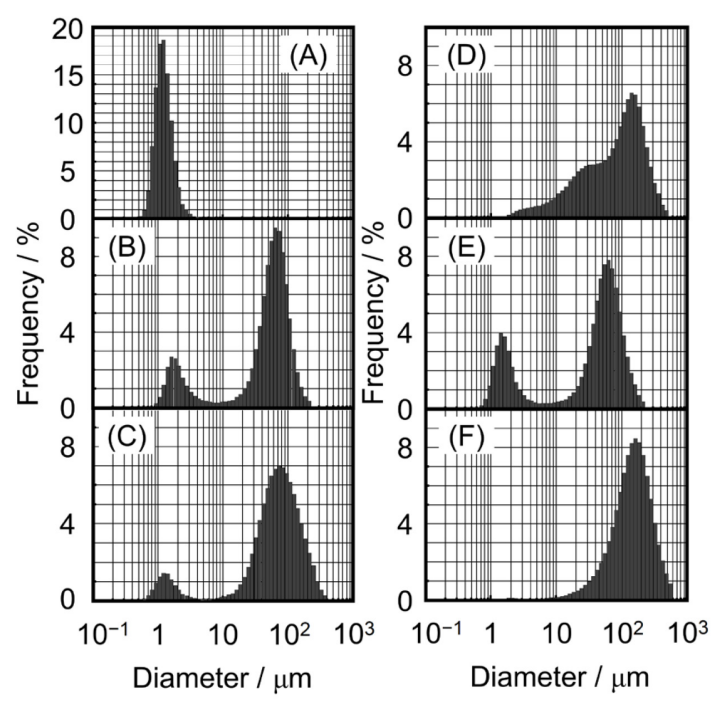

Fig. 2. Size distribution of cells or flocs measured by laser diffraction analysis. (A) P. aeruginosa PAO1, (B) $P$. oryzihabitans AO-363, (C) PAO1+AO-363 (PAO1/AO-363=1/3), (D) B. cereus SM-5, (E) AO-363, (F) SM-5+AO-363 (SM-5/AO-363=1/3).

Table 1. Bacterial flora of microbial flocs.

\begin{tabular}{cc}
\hline \multicolumn{1}{c}{ Strain } & $\begin{array}{c}\text { Biofilm-forming } \\
\text { strain/AO-363 }(\%)\end{array}$ \\
\hline PAO1+AO-363(1:1) & $96 / 4$ \\
PAO1+AO-363(1:3) & $84 / 16$ \\
SM-5+AO-363(1:1) & $75 / 25$ \\
SM-5+AO-363(1:3) & $79 / 21$ \\
\hline
\end{tabular}

bimodal peak probably indicates the coexistence of two types of flocs with different sizes. In the co-culture of SM-5 and AO-363 strain in a $1: 3$ ratio (Fig. 2F), the peak of AO-363 corresponding to its planktonic state was almost completely absent (Fig. 2E). The floc peak of the AO-363 (68 $\mu \mathrm{m})$ appeared to be integrated into a single peak with SM-5 flocs. The increase in the most frequent diameter from 152 to $175 \mu \mathrm{m}$ suggested the formation of the complex microbial flocs.

3.2 Determination of bacterial composition of the microbial flocs

The bacterial composition of the flocs was evaluated by colony counting with a standard dilution plating method. To avoid overlapping colonies, the floc fraction obtained by low-speed centrifugation was diluted to the appropriate density of bacterial cells.

As shown in Table 1, all flocs were determined to be composite flocs consisting of both biofilm-forming bacteria and floc-forming AO-363. Only $16 \%$ of AO-363 cells was detected in flocs after mixing equal volumes of PAO1 $\left(\mathrm{OD}_{600}=0.2\right)$ and $\mathrm{AO}-363 \quad\left(\mathrm{OD}_{600}=0.6\right)$ culture, whereas the bimodal curve of the mixture (Fig. 2C) showed a higher peak of the AO-363 flocs. This is because the number of scatters in a small-diameter peak is much greater than that in a
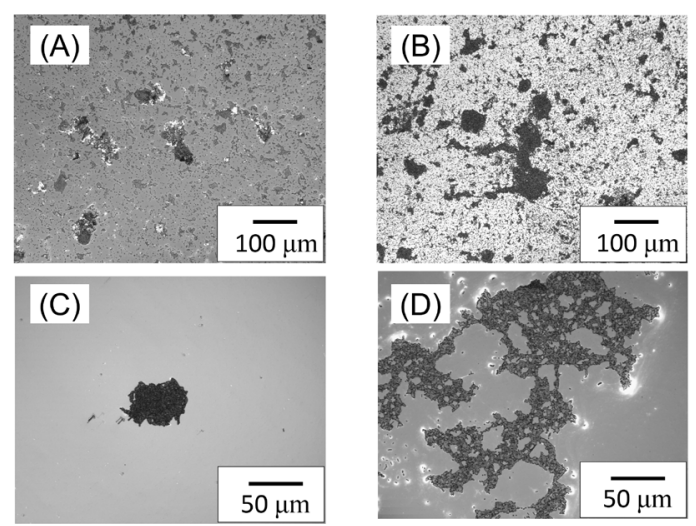

Fig. 3. Photo image of biofilm by confocal laser scanning microscope. (A) PAO1, (B) PAO1+AO-363 (PAO1/AO-363=1/3), (C) B. cereus SM-5, (D) SM-5+AO-363 (PAO1/ AO-363=1/3).
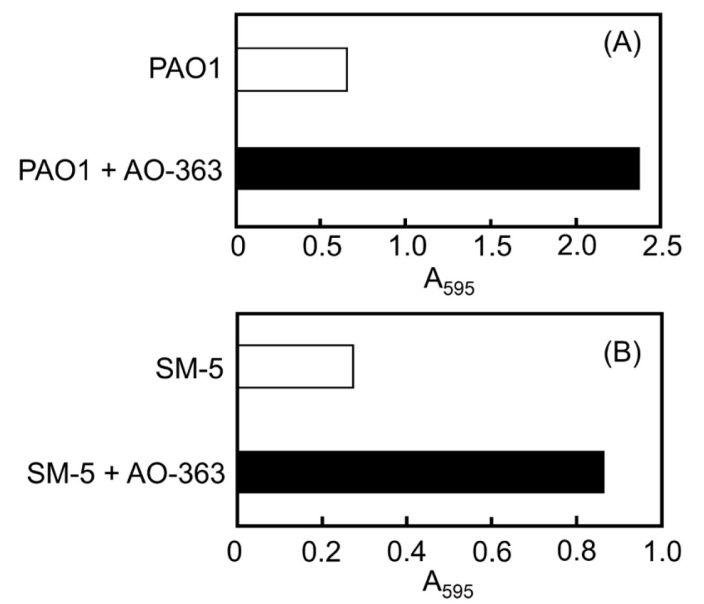

Fig. 4. Quantitative determination of biofilm after culturing for $24 \mathrm{~h}$ at $30^{\circ} \mathrm{C}$. (A) PAO1+AO-363 (PAO1 $/ \mathrm{AO}-363=1 / 3)$, (B) $\mathrm{SM}-5+\mathrm{AO}-363 \quad(\mathrm{SM}-5 / \mathrm{AO}-363=$ $1 / 3)$.

large-diameter peak when the scattering light distribution is given. The abundance ratio of AO-363 in complex flocs with PAO1 increased with increase in the initial inoculation amount of AO-363, but was hardly dependent on the inoculation ratio of AO-363 and SM-5.

3.3 Development of biofilm formation derived from microbial complex flocs

To investigate whether the complex flocs enhanced biofilm formation, culture broth containing composite flocs was incubated in polystyrene wells. After $24 \mathrm{~h}$ of incubation at $30^{\circ} \mathrm{C}, \mathrm{CV}$ solution was added to stain the biofilms for observation with a real color confocal microscope equipped with a xenon lamp (Fig. 3). Two kinds of bacterial cells could be distinguished after CV staining. No difference in morphology was observed between the biofilms formed solely by PAO1 cells (Fig. 3A) and those formed by PAO1 and AO-363 cells (Fig. 3B). On 
the other hand, sparsely adhered cells were observed for SM-5 (Fig. 3C). The biofilm formed solely by SM-5 cells was consisted from the island structure of dense flocs and its approximate diameter was comparable to the floc size observed in Fig. 2D. By flocculation of SM-5 and AO-363, a different morphology of the biofilm having mesh structure was observed (Fig. 3D).

By measuring the absorbance of the eluted CV (A595), the amount of biofilm formed was estimated (Fig. 4). Drastic increase in the $\mathrm{A}_{595}$ was observed for both complex microbial flocs. The biofilm amount formed by the mixture of biofilm-forming and floc-forming bacteria at a ratio of $1: 3$ was 3.7 or 3.2 times greater than that formed by PAO1 or SM-5 monoculture, respectively.

These results suggest that complex flocculation between biofilm- and floc-forming bacteria promotes development of a complex biofilm. By mixing the cultures, the cells of biofilm-forming bacteria could be incorporated into microbial flocs, irrespective of their intrinsic floc-forming ability.

\section{CONCLUSIONS}

Microbial flocs of $P$. oryzihabitans AO-363 successfully trapped the cells of biofilm-forming bacteria, regardless of their own flocculation ability. The relative abundance of AO-363 in flocs increased with an increase in the ratio of AO-363/PAO1 in the mixture, while the abundance of SM-5 and AO-363 was relatively similar in the flocs, even with a 3 -fold excess of AO-363. The amount of biofilm formed by SM-5 and PAO1 in complex microbial flocs was 3-4 times greater than that formed without the floc-forming AO-363. These results suggest that the EPS components forming the flocs were easily incorporated into the biofilms around the micro-colonies and that biofilm formation on the polystyrene surfaces was successfully achieved.

\section{ACKNOWLEDGEMENTS}

We are grateful to Prof. T. Morohoshi for helpful discussions. This work was supported by Core Research for Evolutional Science and Technology-Japan Science and Technology Agency (CREST-JST).

\section{REFERENCES}

[1] H.-C. Flemming and J. Wingender, Nat. Rev. Microbiol., 8, 623-633 (2010).

[2] H.-C. Flemming, J. Wingender, U. Szewzyk, P. Steinberg, S. A. Rice, and S. Kjelleberg, Nat. Rev. Microbiol., 14, 563-575 (2016).

[3] K. Sauer, M. C. Cullen, A. H. Rickard, L. A. H. Zeef, D. G. Davies, and P. Gilbert, $J$. Bacteriol., 186, 7312-7326 (2004).

[4] B. L. Pihlstrom, B. S. Michalowicz, and N. W. Johnson, Lancet, 366, 1809-1820 (2005).

[5] L. Hall-Stoodley and P. Stoodley, Cell. Microbiol., 11, 1034-1043 (2009).
[6] G. Reid, Int. J. Antimicrob. Agents, 11, 223-226 (1999).

[7] M. Herzberg and M. Elimelech, J. Membr. Sci., 295, 11-20 (2007).

[8] B.-K. Hwang, W.-N. Lee, K.-M. Yeon, P.-K. Park, C.-H. Lee, I.-S. Chang, A. Drews, and M. Kraume, Environ. Sci. Tech., 42, 3963-3968 (2008).

[9] Y. Xiong and Y. Liu, Appl. Microbiol. Biotechnol., 86, 825-837 (2010).

[10] M. Hentzer, K. Riedel, T. B. Rasmussen, A. Heydorn, J. B. Andersen, M. R. Parsek, S. A. Rice, L. Eberl, S. Molin, N. Høiby, S. Kjelleberg, and M. Givskov, Microbiology, 148, 87-102 (2002).

[11] R. Singh, D. Paul, and R. K. Jain, Trends Microbiol., 14, 389-397 (2006).

[12] Y. Chen, F. Yan, Y. Chai, H. Liu, R. Kolter, R. Losick, and J.-H. Guo, Environ. Microbiol., 15, 848-864 (2013).

[13] B. Hu, R. Qi, W. An, M. Xu, Y. Zhang, X. Bai, H. Bao, Y. Wen, J. Gu, and M. Yang, Eur. J. Protistol., 49, 491-499 (2013).

[14] B. Q. Liao, D. G. Allen, I. G. Droppo, G. G. Leppard, and S. N. Liss, Water Res., 35, 339-350 (2001).

[15] X. Y. Li and S. F. Yang, Water Res., 41, 1022-1030 (2007).

[16] B.-M. Wilén, B. Jin, and P. Lant, Water Res., 37, 2127-2139 (2003).

[17] H. Salehizadeh and S. A. Shojaosadati, Biotechnol. Advances, 19, 371-385 (2001).

[18] F. F. Dias and J. V. Bhat, Appl. Microbiol., 12, 412-417 (1964).

[19] C. Ryder, M. Byrd, and D. J. Wozniak, Curr. Opin. Microbiol., 10, 644-648 (2007).

[20] K. Pedersen, Appl. Environ. Microbiol., 43, 6-13 (1982).

(Received April 26, 2017; Accepted May 8, 2017; Published Online June 1, 2017) 